\title{
FATIGUE ASSESSMENT OF A SEMI-TRAILER USING FE SPECTRAL ANALYSIS COMBINED WITH RANDOM FATIGUE CALCULATION
}

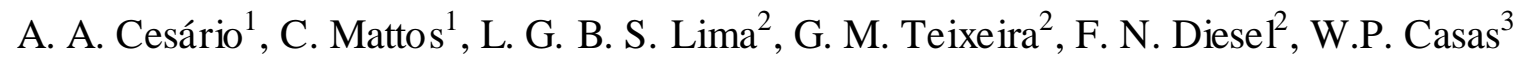 \\ ${ }^{1}$ Guerra S/A Implementos Rodoviários (andre.cesario@guerra.com.br) \\ ${ }^{2}$ Engineering Simulation Scientific Software - ESSS \\ ${ }^{3}$ Grupo de Mecânica Aplicada, Departamento de Engenharia Mecânica, Universidade Federal \\ do Rio Grande do Sul - UFRGS
}

\begin{abstract}
In this work a numerical methodology is proposed to assess the fatigue failure of a semi-trailer under random load. This methodology combines PSD (Power Spectral Density) and FEA (Finite Element Analysis) with equations proposed by Dirlik to evaluate the damage on structures. Instead of using time domain analysis, as the traditional methodologies, a frequency domain analysis is adopted. This approach uses the PSD's of the imposed loads, which are curves of load in frequency domain. Using well-known experimental techniques, the acceleration of the structure is obtained with accelerometers positioned at the suspension region of the semi-trailer, while the strains are obtained with strain gages positioned in critical points of the structure. The measured accelerations are used as input data of the Finite Element Analysis in order to get the PSD of stresses. The experimental strains are used to calibrate and validate de numerical model. The most important advantage of the proposed methodology is its lower computational cost compared with the time domain analysis. This approach seems to be more appropriate mainly in cases of non-deterministic loads, even though its content is statistically constant. Then, with the pertinent PSD of stresses at each node, a PSD cycle counting is performed and combined with the fatigue curve of the material in order to get the cumulative damage according to the Miner Rule. It should be mentioned, however, that this methodology is valid considering the hypothesis of a linear elastic analysis.
\end{abstract}

Keywords: Fatigue, Frequency Domain, Dirlik's Approach.

\section{INTRODUÇÃO}

Currently, $58 \%$ of cargo transported in Brazil, are made by road transportation, compared to $25 \%$ of the railroad. Excluding the transportation of iron ore, which occurs by rail, highways respond for more than $70 \%$ of cargo transported [1].

Although the highways are widely used in Brazil, the country presents deficiencies in road infrastructure, which suggests a paradox. $1581104 \mathrm{~km}$ of highways in Brazil, only 
$213909 \mathrm{~km}$ are paved, which represents $13.5 \%$ of the mesh. Of this total, over $15,000 \mathrm{~km}$ of highways granted [2].

The most complete and updated analys is work of the brazilian highways was carried out by National Confederation of Transport in 2011. This study evaluated a total of 92747 $\mathrm{km}$, including all federal roads paved and the most significant sections of state highways. These studies showed that $47.9 \%$ had bad pavement, and of these $14 \%$ have pavement in precarious condition [2].

Inserted in this context are the road implement, vehicles responsible for the specific function of cargo transportation on the highways.

When it comes exclusively of fuels transportation, $100 \%$ of the transport between the distributors bases and consumers, are carried by road via tank-type road implement. The transport between the primary and secondary basis of distribution is accomplished primarily by rail, but $31 \%$ of the total also use the road, although this is not the most economically efficient to overcome great distances [3].

According to what was exposed, the correct prediction of the fatigue life of these implements it is as fundamental as complex, since the transport of fuel need to be safe and agile, even on bad roads of Brazil. For this reason, a road implement type Bitrem (Fig. 1) tank will be the object of the present study.

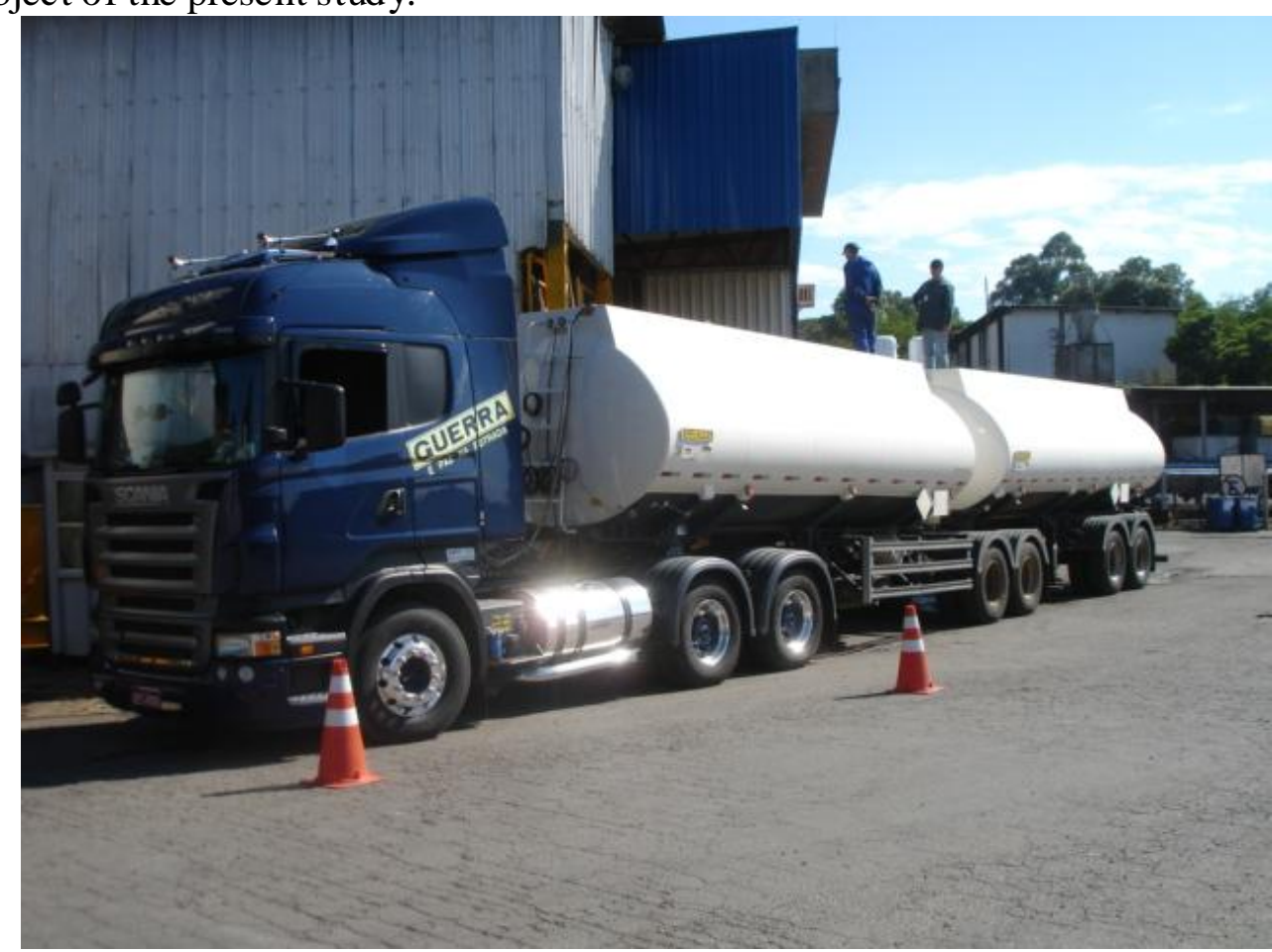

Figure 1. Road implement study object of this paper.

\section{METHODOLOGY}

Through the use of CAE tools, it is possible to estimate the fatigue life of vehicles without the fabrication of prototypes or with fewer of these, which has as its main advantages in reducing time and costs involved in the project. 
The estimated life of a vehicle can be addressed by two main methods: the time domain or frequency domain. The methodology of analysis in time domain is the most traditional and considers both the loads and responses expressed in terms of time histories. In the frequency domain loads and responses are expressed statistically by PSDs (Power Spectrum Density).

The physical data can be classified as a deterministic or random. The deterministic data are those that can be found through an explicit mathematical relationship, while the random data are defined by their statistical properties. The random data can be classified according to Fig 2. [4].

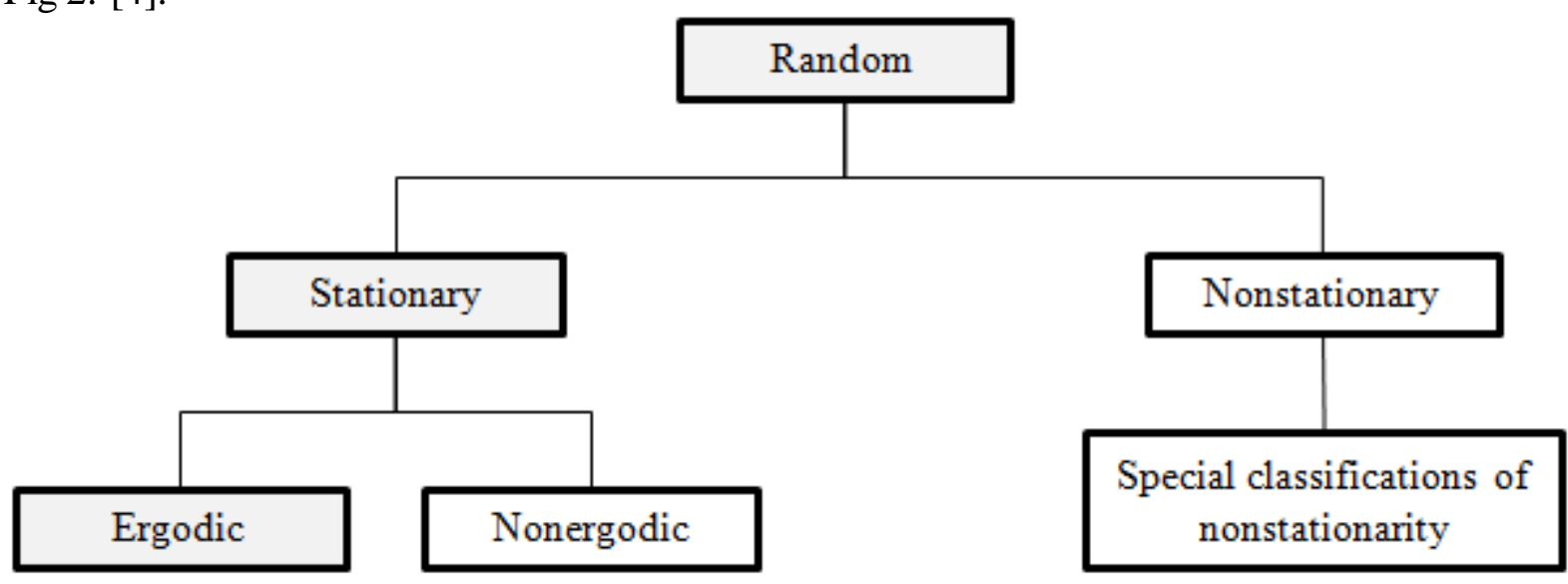

Figure 2. Random data classification.

The track profiles, which correspond to dynamic excitations imposed on the structure of the road implement used in this study, are classified as random, stationary, ergodic and Gaussian processes, according to [5], [6], [7], [8] .

The first step in fatigue life assessment of a road implement is obtain the excitations which are acting in the structure when it is in operation. In frequency domain, the loads due the road roughness are defined in terms of PSD's and CSD's. The PSDs correspond to each point of load of the road implement structure, while the CSDs (Cross Spectral Density) are responsable by correlation between the PSDs allowing the phase differences between the excitations are considered.

Several authors have developed mathematical models of PSDs tracks, among which stand out: Cebon, Gillespie, Houlbolt e Gauss. Another way to obtain such excitations is through instrumentation and accelerations data collection directly from the road implement, method that was used in this work.

Once known the acceleration PSDs and CSDs values, one can find the PSDs response of the structure, given in terms of stress. These are obtained by the use of linear transfer function, by means of a frequency response analysis. Of course, the system is treated as linear.

The accumulated damage to the structure can be determined using the rule-Miner Palmgren, by use of spectral moments extracted directly from the PSDs of stress. Fig. 3 shows the flowchart for analysis. 


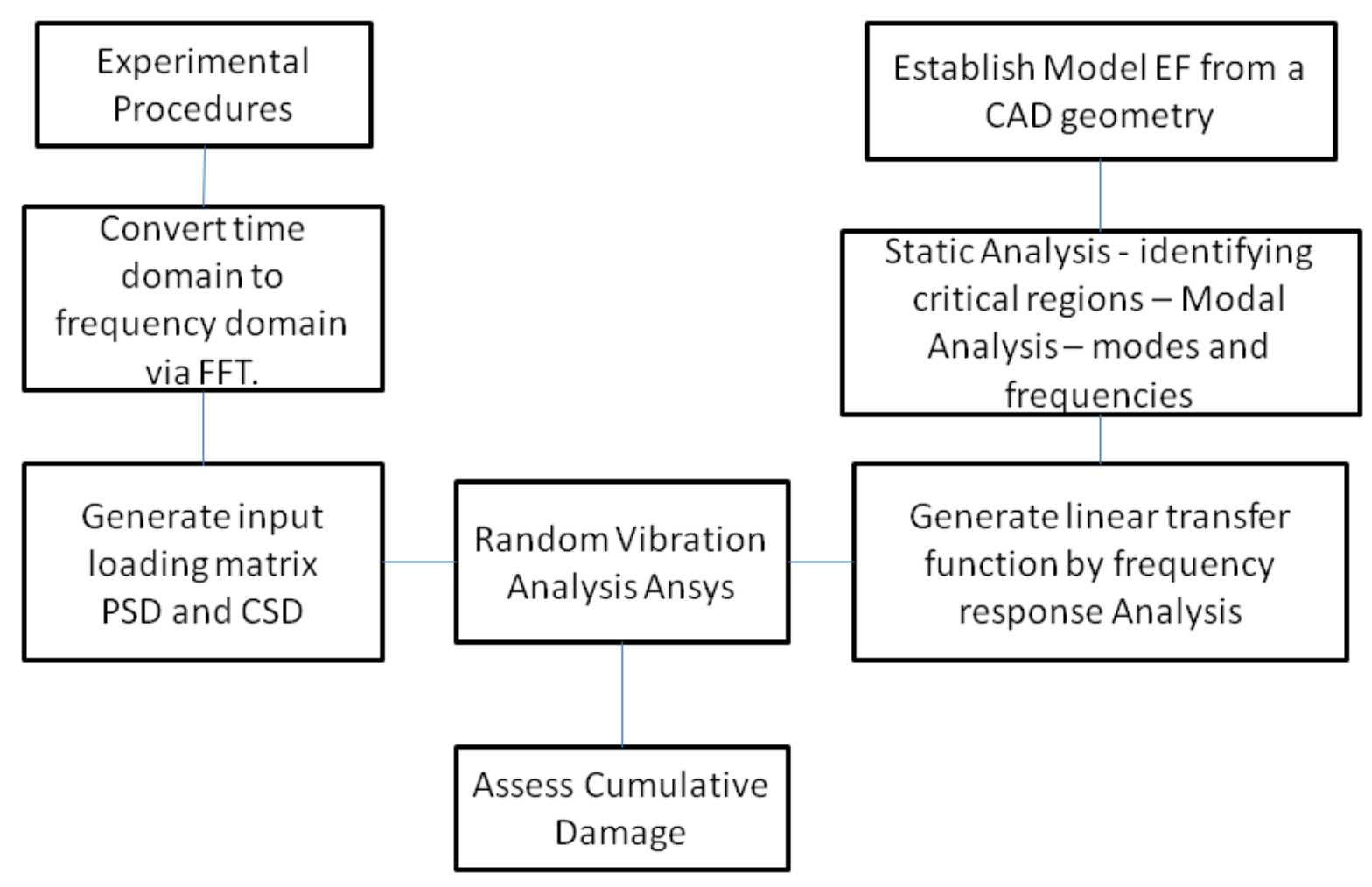

Figure 3. Analysis flowchart.

\subsection{Experime ntal Procedures}

The implement road was instrumented in order to obtain experimentally the dynamic loads to which it is submitted. For this, seven accelerometers were used, positioned in the road implement structure, being six in the region of the suspension and in the kingpin region, as shown in Fig 4.

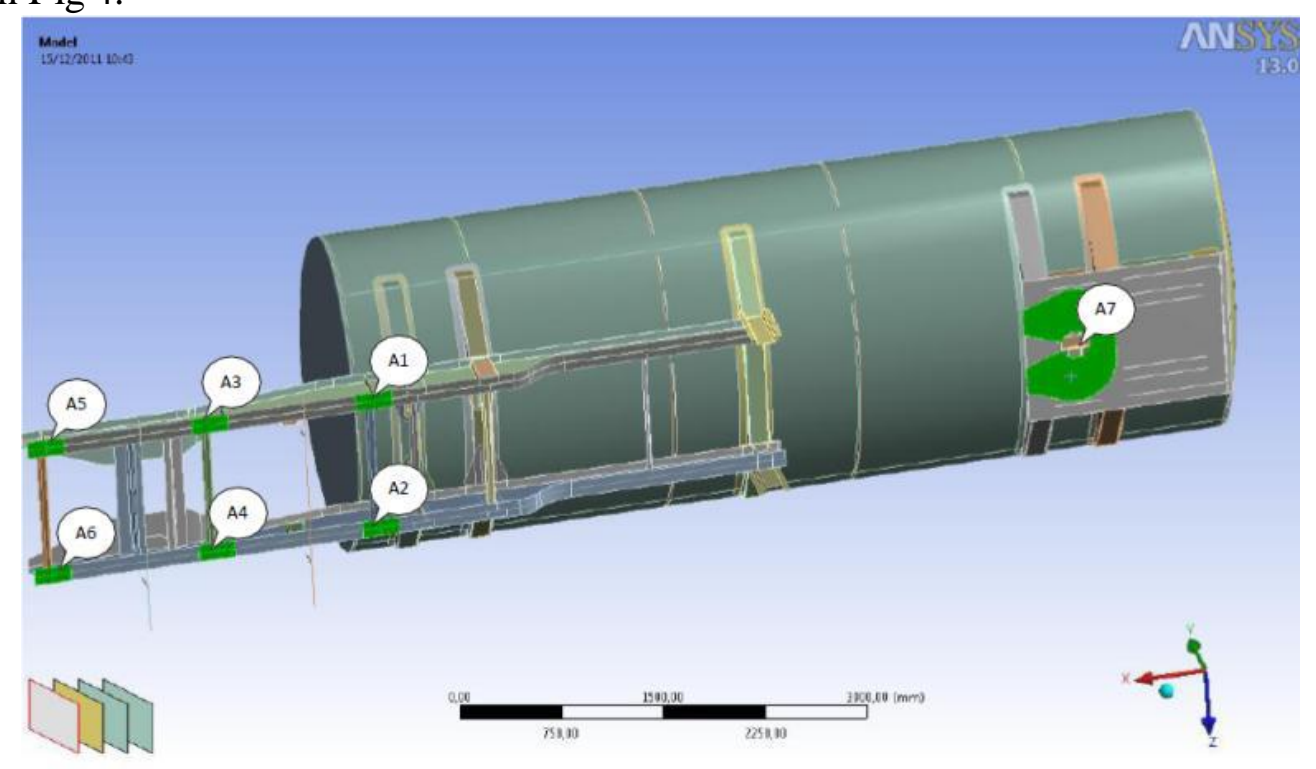

Figure 4. The seven accelerometers positioned in the road implement. 
The largest effects on the fatigue life of the implement are caused by the vertical dynamics, so the 7 capacitive accelerometers Silicon Designs model 2210-025 (Fig. 5) were positioned in order to measure the vertical accelerations coming from the horizontal displacement of the implement running by roads with different roughness profiles. The accelerometers are capable of measuring $+/-25 \mathrm{~g}$.

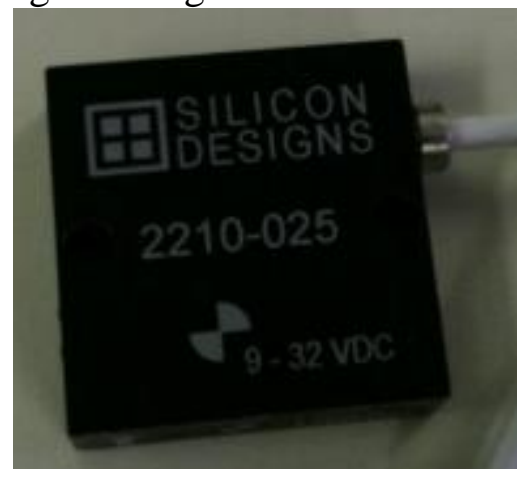

Figure 5. Model of accelerometer used in experimental procedures.

According to the Nyquist frequency, the sampling frequency must be at least equal to twice the highest frequency to be measured. If the sampling frequency is to infer the Nyquist frequency, the phenomenon known as aliasing occurs, shown in Figure 6. [4], [5] e [6].

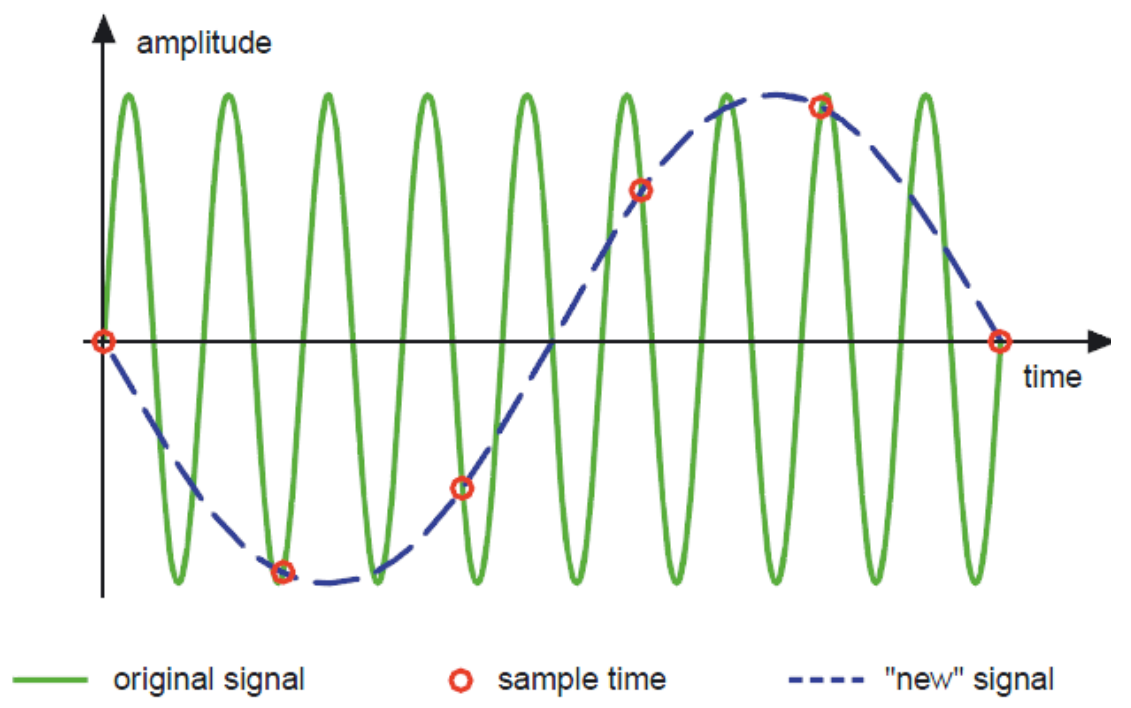

Figure 6. Aliasing effect.

Therefore, to ensure correct measurement of excitations at frequencies up to $300 \mathrm{~Hz}$ and avoid to generate redundant data, was used a sampling frequency of $600 \mathrm{~Hz}$ in the acquisition of the signals.

In addition to the acceleration in the seven points of the Bitrem also be experimentally determined the resulting stress at certain points of the structure for use as calibration of the numerical model. For collect this data were used triaxial Kyowa strain gages $\left(0^{\circ} / 45^{\circ} / 90^{\circ}\right)$ rosette type with $6 \mathrm{~mm}$, as show in Fig. 7. 

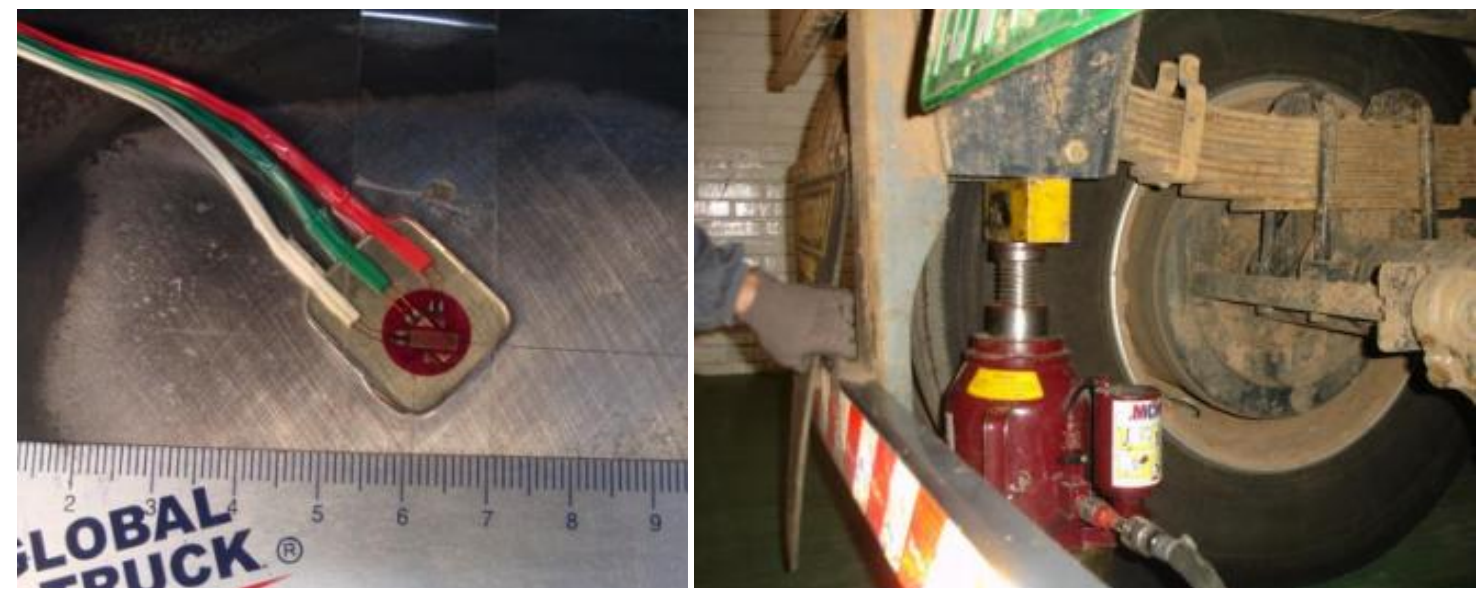

Figure 7. Strain Gages used to calibrate the numerical model.

When quantities collected in the time domain are converted to the frequency domain, one of the problems that must be avoided is the phenomenon known as spectral leakage. This phenomenon occurs when the beginning and end of sampling are performed so sudden, occurring a "leakage" of power to adjacent frequencies. The Fig. 8 shows an example of spectral leakage [4], [5] e [6].
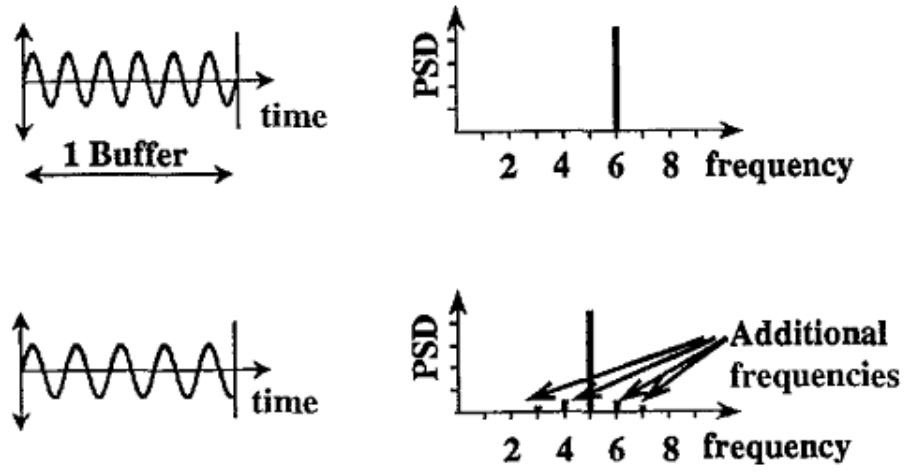

Figure 8. Spectral leakage phenomenon.

To reduce this phenomenon, it is indicated using the data window, which ensures smooth at the beginning and end of a data block. There are several types of windows, but there is not one that is suitable for all situations. There is a consensus, however, about the non-use of the same. In the true, the fact of not using the data window means the same as use a rectangular window, which is exactly the least indicated, by causing abrupt beginning and end of the data window. [4], [5] e [6].

Among the main windows, some are more simple to implement and have good results, such as: Hamming, Von Hann (Hanning), Kaiser-Bessel e Blackman - Harris. In this work we opted for a Hanning window type, given by Eq. (1):

$$
w(n)=0,5-0,5 \cos \left(\frac{2 \pi n}{N}\right)
$$

where:

$n$ is the number of point data. 


\subsection{Conve rsion of data tracks in PSD and generation of matrix loading}

The acceleration signals collected in the time domain must be transformed into acceleration PSDs and CSDs. The mathematical operation that allows this transformation is the Fourier transform. For a random, stationary and ergodic process, the analysis of a single record of the time signal allows to obtain the statistical properties of the entire process. The average time of a random variable $x(t)$ is equal to the expected value of $x(t)$ according to Eq. (2) [4] e [6].

$$
E[x(t)]=\lim _{T \rightarrow \infty} \frac{1}{T} \int_{0}^{T} x(t) d t
$$

The autocorrelation function which indicates how a signal is correlated with itself, at different instants of time, for a random and stationary process is given by:

$$
R_{x}(\tau)=E[x(t) x(t+\tau)]=\lim _{T \rightarrow \infty} \int_{0}^{T} x(t) x(t+\tau) d t
$$

The cross-correlation function of two sets of random data describes the dependence of the sets of data relative to each other, i.e., describes how two processes are correlated. The cross-correlation function for random and stationary process is given by Eq. (4):

$$
R_{x y}(\tau)=E[x(t) y(t+\tau)]=\lim _{T \rightarrow \infty} \int_{0}^{T} x(t) y(t+\tau) d t
$$

For a random and stationary process the PSD is given by the Fourier transform of the autocorrelation function:

$$
S_{x}(w)=\int_{-\infty}^{\infty} R_{x}(\tau) e^{-j w \tau} d \tau
$$

where:

$\omega=2 \pi f$

The CSD can be obtained similarly by the Fourier transform of the cross-correlation function:

$$
S_{x y}(\omega)=\int_{-\infty}^{\infty} R_{x y}(\tau) e^{-j \omega \tau} d \tau
$$

The Eqs. (5) e (6) are valid in the range from $-\infty \leq f \leq \infty$, ie, for a two-sided bilateral process. For structural problems, however, the negative frequencies has physical sense and so, only the positive frequencies should to be used, given by the one-sided PSD e CSD which are 
valid in the range from $0 \leq f \leq \infty$ according to Eqs. (7) e (8):

$$
\begin{gathered}
G_{x}(f)=2 S_{x}(f) \\
G_{x y}(f)=2 S_{x y}(f)
\end{gathered}
$$

As the cross-correlation function is not an even function, CSD is a complex number and generally may be represented by:

$$
G_{x y}(f)=C_{x y}(f)-j Q_{x y}(f)
$$

where:

$C_{x y}(f)$ is called coincident spectral density function (co-spectrum);

$Q_{x y}(f)$ is called quadrature spectral density function (quad-spectrum).

The collected experimentally data are discrete time records. (Fig. 9). When these data are classified as random and stationary processes, one can estimate the CSD PSD directly from the original data, using the Fast Fourier Transform (FFT). The FFT is an efficient algorithm for calculating the discrete Fourier transform (DFT). From the computational point of view, the use of FFT is advantageous when compared to the previous method, i.e. the PSD estimate and CSD through the Fourier transform of the functions of autocorrelation and crosscorrelation, respectively.

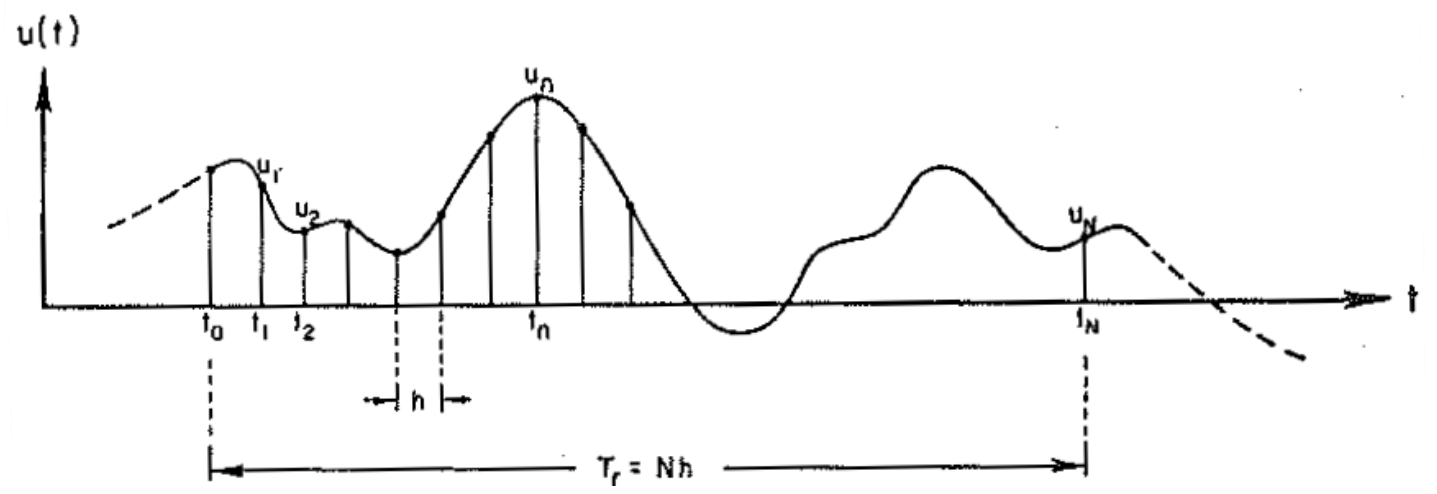

Figure 9. Discrete experimental data.

Assuming that the random variable $x(t)$ is sampled at $N$ points evenly spaced points, $h$, according to Fig. 8, a FFT é given by:

$$
X(f, T)=h \sum_{n=0}^{N-1} x_{n} e^{-j 2 \pi f n h}
$$

where:

$x_{n}=x(n h) \quad n=0,1,2, \ldots \mathrm{N}-1$

The discrete values of frequencies are given by: 


$$
f_{k}=k f_{0}=\frac{k}{T}=\frac{k}{N h}
$$

where:

$k=0,1,2, \ldots, \mathrm{N}-1$

At these frequencies, the FFT is given by:

$$
X_{k}=\frac{X\left(f_{k}, T\right)}{h}=\sum_{n=0}^{N-1} x_{n} e^{\left(-j \frac{2 \pi k n}{N}\right)}
$$

It should be noted, however, the results have unique values only until $\mathrm{N} / 2$, which corresponds to the point where the cutting occurs at the Nyquist frequency.

Thus, the PSDs and CSDs can be estimated by FFT according to Eqs. (13) e (14), respectively [4]:

$$
\begin{gathered}
\tilde{G}_{k}(f)=\frac{2 h}{N}\left|X_{k}\right|^{2} . \\
\tilde{G}_{x y}\left(f_{k}\right)=\frac{2 h}{N}\left|X_{k}^{*} Y_{k}\right| .
\end{gathered}
$$

where:

$X_{k} *$ is the complex conjugate of $X k$.

For multiple inputs, the loading matrix, comprising PSDs and CSDs, is of the form:

$$
\tilde{G}_{x y}\left(f_{k}\right)=\left[\begin{array}{cccc}
\tilde{G}_{11}(\omega) & C_{12}(\omega)+j Q_{12}(\omega) & C_{13}(\omega)+j Q_{13}(\omega) & \ldots \\
C_{12}(\omega)-j Q_{12}(\omega) & \tilde{G}_{22}(\omega) & C_{23}(\omega)+j Q_{23}(\omega) & \ldots \\
C_{13}(\omega)-j Q_{13}(\omega) & C_{23}(\omega)-j Q_{23}(\omega) & \tilde{G}_{33}(\omega) & \ldots \\
\vdots & \vdots & \vdots &
\end{array}\right]
$$

\subsection{Generation of Transfer Functions}

Having set up the input loading matrix in terms of PSDs and CSDs, the next step is to determine the transfer function of the structure for, subsequently, be possible to calculate the stress PSD. The transfer function can be defined experimentally or by a frequency response analysis directly through the Ansys Workbench . To this end, applies a series of sinusoidal unit loads in the structure and the response for each frequency is obtained. [5]

The equation of motion for a structure can be given by:

$$
[M] \ddot{x}(t)+[C] \dot{x}(t)+[K] x(t)=f(t)
$$

where: 
$[M]$ is the global mass matrix;

$[C]$ is the global damping matrix;

$[K]$ is the global stiffness matrix;

$x(t)$ is the vector of nodal displacements;

$f(t)$ is the vector of nodal forces.

For a sinusoidal time- varying load, the displacement vector also present sinusoidal variatio. Thus, $f(t)$ and $x(t)$ are [7]:

$$
\begin{aligned}
& f(t)=F \cdot e^{i w t} \\
& x(t)=X \cdot e^{i w t}
\end{aligned}
$$

Differentiating displacement with respect to time yields expressions for the velocity and acceleration of the component:

$$
\begin{aligned}
& \dot{x}(t)=i \cdot \omega \cdot X \cdot e^{i \omega t} \\
& \ddot{x}(t)=-\omega^{2} \cdot X \cdot e^{i \omega t}
\end{aligned}
$$

Substituting Eq. (19) and (20) in Eq. (16), after some mathematical manipulation, we find:

$$
X(\omega)=H(\omega) \cdot F(\omega)
$$

where:

$H(\omega)$ is the linear transfer function, which is given by:

$$
H(\omega)=\left[-[M] \cdot \omega^{2}+[C] \cdot i \cdot \omega+[K]\right]^{-1}
$$

$X(\omega)$ is the vector of displacement amplitude;

$F(\omega)$ is the vector of force amplitude.

For the units of PSD analysis remain consistent, the terms of transfer function, now presented as a function of frequency, $f$, must be squared. Thus, the PSD of stress $G(f)$ for a unique input load $W(f)$ can be given by [5] e[7]:

$$
G(f)=|H(f)|^{2} \cdot W(f)
$$

For multiple loads applied simultaneously, the Eq. (23) can be given by [7]:

$$
G(f)=\sum_{a=1}^{n} \sum_{b=1}^{n} H_{a}(f) H_{b}{ }^{*}(f) \tilde{G}_{a b}(f)
$$

where:

$n$ is the number of simultaneous loading inputs: 


\subsection{Fatigue Analysis in the Frequency Domain}

The damage caused by fatigue in structures is calculated in the frequency domain, based on statistical properties of the PSDs response. These properties can be determined through the spectral moments, which are calculated according to Eq. (25) (Fig. 10) [5], [6], [7] e [8]:

$$
m_{n}=\int f^{n} \cdot G(f) \cdot d f, \operatorname{com} n=1, \ldots, 4
$$

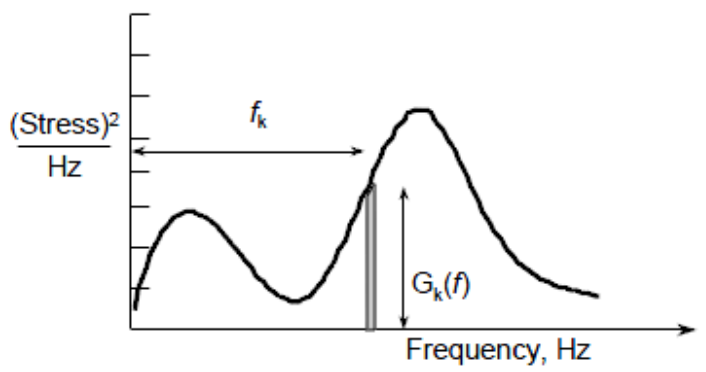

Figure 10. Method of calculation of spectral moments.

The value called expected of peaks $E[P]$ is given by:

$$
E[P]=\sqrt{\frac{m_{4}}{m_{2}}}
$$

Whereas high cycle fatigue $(\mathrm{S}-\mathrm{N})$, the curve of the material, which relates the amplitudes of the alternating stress $S_{A}$ and the number of cycles to failure, $N$ may be determined in simplified form as follows [8]:

$$
B=N S_{A}^{m}
$$

where:

$B$ and $m$ are materials properties.

The damage accumulated by random loads can be evaluated through the rule of linear damage accumulation Palmgren-Miner, as follows [8]:

$$
E[A D]=\int_{0}^{\infty} \frac{n\left(S_{A}\right)}{N\left(S_{A}\right)} d S_{A}=\int_{0}^{\infty} \frac{p\left(S_{A}\right) N}{N\left(S_{A}\right)} d S_{A}
$$

where:

$n\left(S_{A}\right)$ is the number of cycles applied at stress amplitude level $S_{A}$;

$p\left(S_{A}\right)$ is the the probability density function (PDF) of the stress amplitude $S_{A}$.

Through the PDF one can determine the probability of a certain stress range occur in a certain interval. For example, according to Fig. 10, the probability of the stress range occurs between $S i$ - $d s / 2$ e $S i$ - $d s / 2$ is given by $p(S i) . d s$ [5]. 


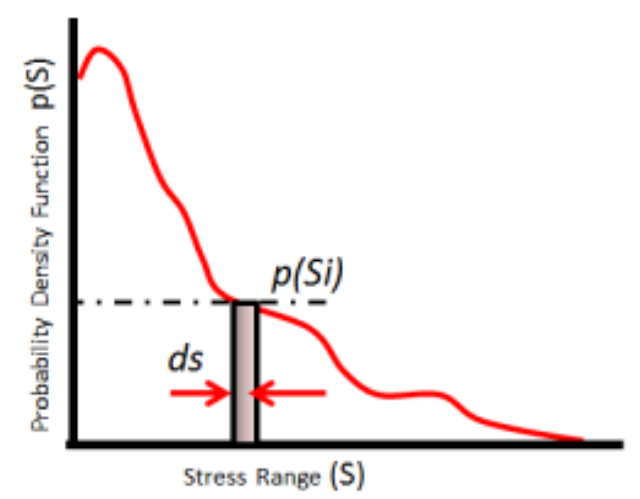

Figure 11. Probability Density Function.

Substituting Eq. (27) in Eq. (28), results in a general equation for the accumulated damage calculation under random loading [8]:

$$
E[A D]=\frac{T \cdot E[P]}{B} \int_{0}^{\infty} S_{A}^{m} p\left(S_{A}\right) d S_{A}
$$

where:

$T$ is the duration time of random loading.

Several authors have developed models based on Eq. (29) using different definitions of the probability density function $p\left(S_{A}\right)[8]$.

The narrow band model proposed Bendat is the first model to estimate the fatigue damage in the frequency domain, however, presents results conservative. To address this problem, some researchers have de veloped other models in order to make the results closer to reality. The best known are: Wirsching, Tunna, Hancock and Chaudhury and Dover.

Dirlik method presented a closed form solution developed from Monte Carlo simulations. According to [5], [7] and [9] this solution is the one that presents more accurate results and therefore was used in this work. The PDF equation proposed by Dirlik is given by:

$$
p\left(S_{A}\right)=\frac{\frac{D_{1}}{Q} \cdot e^{\frac{-Z}{Q}}+\frac{D_{2}}{R^{2}} \cdot e^{\frac{-Z^{2}}{2 \cdot R^{2}}}+D_{3} \cdot Z \cdot e^{\frac{-Z^{2}}{2}}}{2 \cdot \sqrt{m_{0}}}
$$

where:

$$
\begin{gathered}
\gamma=\frac{m_{2}}{\sqrt{m_{0} \cdot m_{4}}} \\
x_{m}=\frac{m_{1}}{m_{0}} \cdot \sqrt{\frac{m_{2}}{m_{4}}}
\end{gathered}
$$




$$
\begin{gathered}
D_{1}=\frac{2 \cdot\left(x_{m}-\gamma^{2}\right)}{1+\gamma^{2}} \cdot \gamma^{2} \\
D_{2}=\frac{1-\gamma-D_{1}+D_{1}^{2}}{1-R} \\
D_{3}=1-D_{1}-D_{2} \\
Z=\frac{S}{2 \cdot \sqrt{m_{0}}} \\
Q=\frac{1,25 \cdot\left(\gamma-D_{3}-D_{2} \cdot R\right)}{D_{1}}
\end{gathered}
$$

\section{COMPUTATIONAL MODEL AND RESULTS}

The accelerations imposed on the road implement were taken when it was moving on dirt roads. The choice of this type of track is due to the greater severity and consequently greater damage that this type of track concerned to the road implement. The Fig. 12 shows a stretch of track where the accelerations were collected.

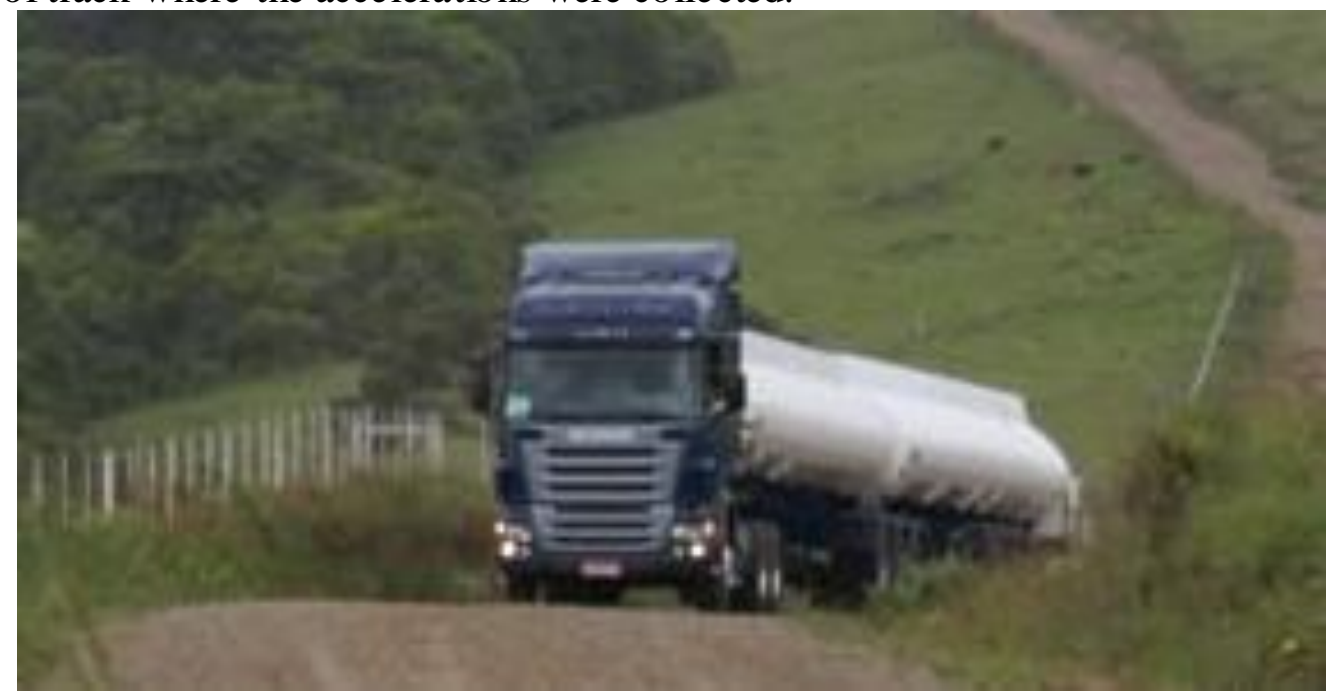

Figure 12. Road implement in test.

From a 3D CAD model of the road implement in study, one FE mesh was obtained in in ANSYS software, as show Figure 13. 


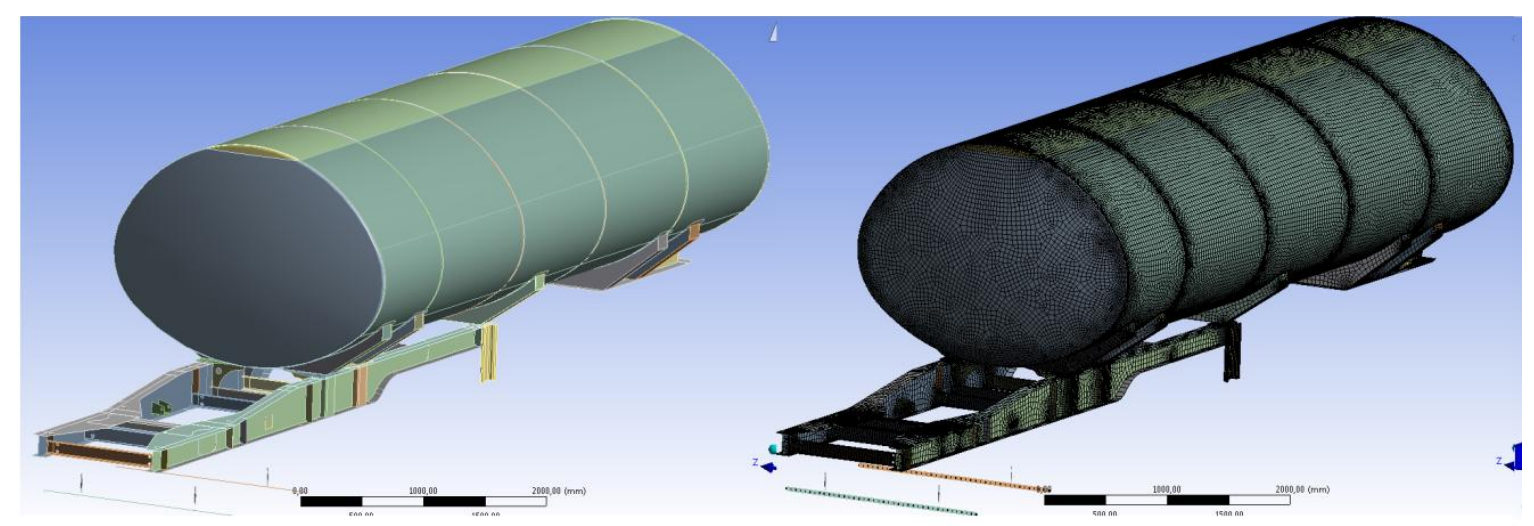

Figure 13. 3D CAD and FE mesh.

As a result of instrumentation, time is obtained historical acceleration for each of seven accelerometers used. These signals are smoothed using a Hanning window and, subsequently transformed into PSD's in frequency domain by the FFT. The Fig. 14 shows signal in time domain of the accelerometer 1 (Fig.4) after the use of Hanning window and the corresponding acceleration PSD.
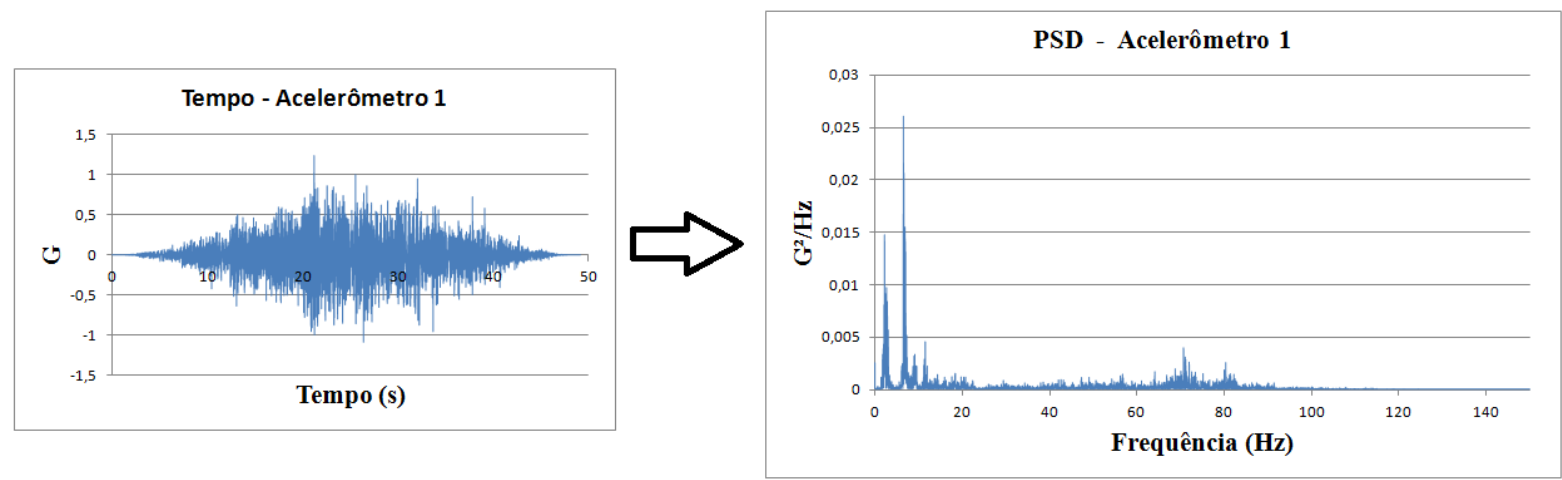

Figure 14. Time domain signal after Hanning and respective PSD.

The fatigue life was defined for a node belonging to the critical region of the road implement. To find this region, a static analysis was carried out where was obtained and the Von Mises stress (Fig. 15), showing the critical region in red. Noteworthy is the mesh refinement in the region of interest, aiming to produce most reliable results. 


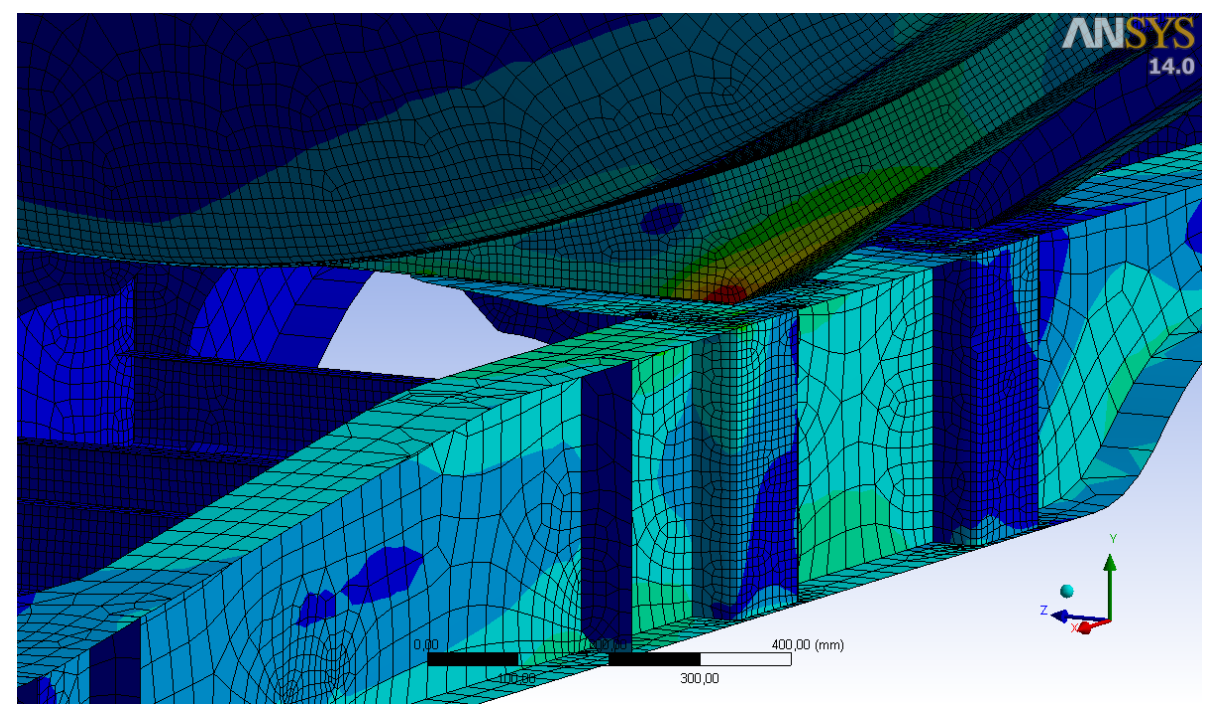

Figure 15. Função Densidade de Probabilidade.

Once known the critical region, we estimated the PSD stress to a node belonging to this and, subsequently, the fatigue life to the same node. The response of the structure is calculated by the method of modal superposition, so, first defined the modes and natural frequencies of vibration of the structure. Independently, the linear transfer function of the structure was determined as has already been defined in Eq. 22 .

In the sequence, a random vibration analysis, was carried out, considering as input the square matrix $7 \times 7$ formed by PSDs and CSDs of the seven collected accelerations signals according to Eq. (15), resulting in the stress PSD. The Fig. 16 shows the results for the stress in the y direction.

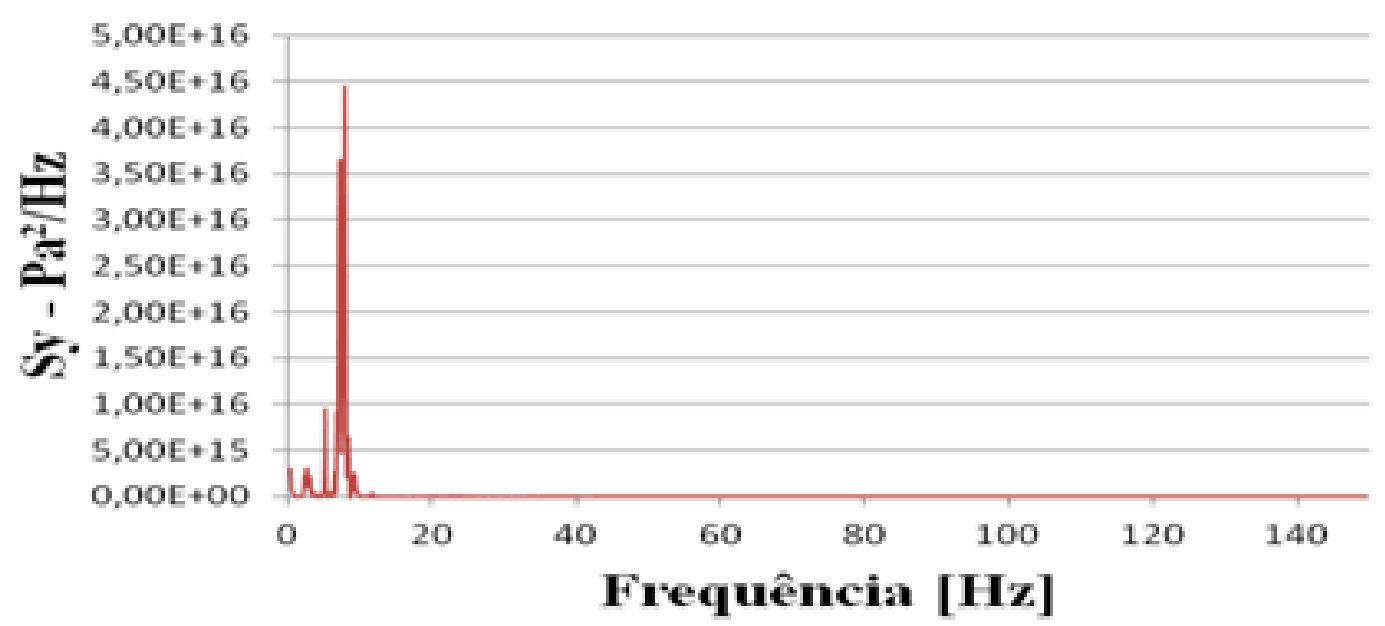

Figure 16. PSD stress in y direction for a node in the critical region.

The above PSD curve obtained was then used to calculate the spectral moments, and the results are listed below:

$$
\begin{aligned}
& \mathrm{m}_{0}=3,724 \cdot 10^{11} \\
& \mathrm{~m}_{1}=1,984 \cdot 10^{12}
\end{aligned}
$$




$$
\begin{aligned}
& \mathrm{m}_{2}=1,436 \cdot 10^{13} \\
& \mathrm{~m}_{4}=1,443 \cdot 10^{15}
\end{aligned}
$$

These spectral moments were the used in Eqs. (31), allowing the calculation of the probability density function, $p\left(S_{A}\right)$, according to Eq. (30).

Finally, the curve $p\left(S_{A}\right)$ was used in the calculation of accumulated damage in the structure by means of Eqs. (26) e (29) and considering B $=9,81 \mathrm{E}+25, \mathrm{~m}=9,09$, data obtained from the curve S-N (Fig. 17) from the plate material of critical region and $\mathrm{T}=7,51 \mathrm{E}+$ 6 seconds (obtained when considering the use of the implement for 8 hours a day, five days per week for a year), resulting in:

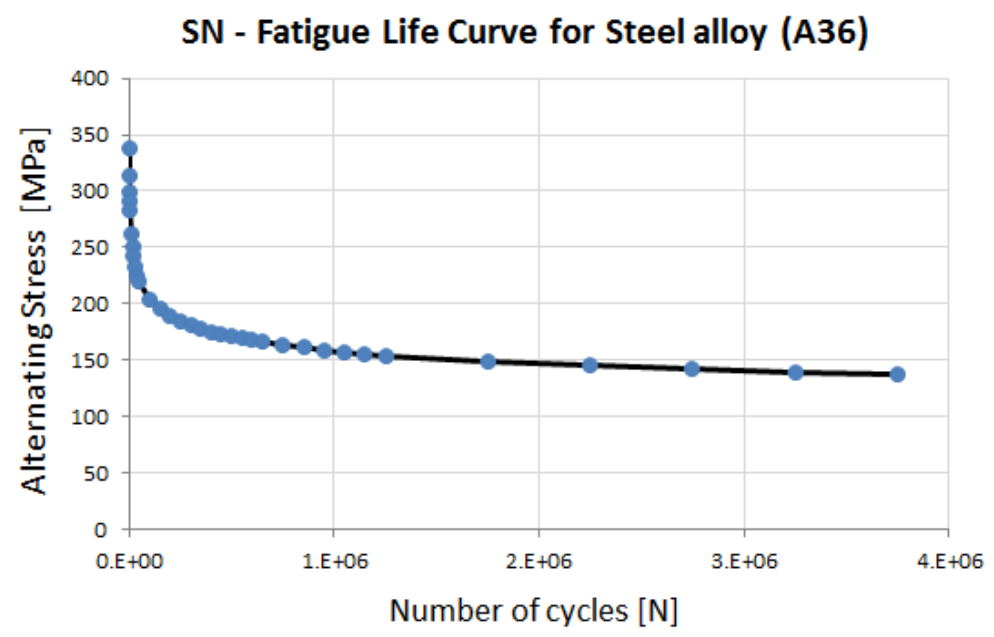

Figure 17. S - N curve of material.

$\mathrm{E}[\mathrm{D}]=0,517$

Or, in other words, a life of 1.933 years, under the conditions described above.

\section{CONCLUSÕES}

In this paper, was proposed a numerical methodology of study of fatigue in frequency domain for a road implement subjected to accelerations experimentally measured on the track and used as input data for numerical model.

One of the main advantages of using this methodology is the reduction of computational time compared to what would be observed when performing an analysis in time domain. At this sense, the process proved to be very fast and intuitive.

The method also allows a better understanding of the dynamic behavior of the structure, because this has answers in the frequency domain.

The results indicate the occurrence of damage in a relatively short period of use of the implement - about half the average time required for verification of actual component failures. This result may be explained by using only a stress component in the calculation of the PSD curve for the region chosen for analysis, just the highest intensity, rather than an equivalent stress, maximum principal stress, or consideration of the critical plane criteria for multiaxial 
stress. Moreover, the proposed method does not consider the strains means for calculating fatigue, which are predominantly compressive in this case.

Finally, the track used in the experimental measurements showed very unfavorable operating conditions, which would probably not be observed during the whole operation of the implement within considered.

For future work we intend to validate the methodology proposed in this paper, with the established methods for estimating the fatigue damage in the time domain. Using a criterion of correctness for the consideration of mean stresses and strains in multiaxial frequency are also needed.

Since the method is validated, is intended to estimate the fatigue life on roads with different roughness profiles and the combination of different profiles, in accordance with the use of each type of road attachment.

\section{REFERENCES}

[1] IPEA 2011, “Gargalos e Demandas da Infraestrutura Rodoviária e os Investimentos do PAC: Mapeamento IPEA de obras rodoviárias", Instituto de Pesquisa Econômica Aplica$d a$, disponível em http://www.ipea.gov.br/sites/000/2/publicacoes/tds/TD_1592_web.pdf, acesso em 29/03/2012.

[2] CNT 2011, "Pesquisa CNT de Rodovias 2011", Confederação Nacional do Transporte, 2011 , disponível em http://pesquisarodovias.cnt.org.br/Documents/PDFs/Principais_dados_PCR2011-1.pdf, acesso em 28/03/2012.

[3] Figueiredo R. "Gargalos Logísticos na Distribuição de Combustíveis Brasileira”, 2006, disponível http://www.forumlogistica.net/site/new/art_Gargalos_Logisticos_na_dist_de_combust.pdf , acesso em 28/03/2012.

[4] Bendat J.S., Piersol A. G., "Randon Data: Analysis and Measurement Procesures". John Wiley \& Sons, New York, 1971.

[5] Bishop N.W.M., "Vibration Fatigue Analysis in the Finite Element Environment", $X V I$ Encuentro del Grupo Español de Fractura, 1999.

[6] Newland D. E., "An Introduction to Random Vibrations, Spectral and Wavelet Analysis", 3 ed., John Wiley \& Sons, New York, 1993.

[7] Halfpenny A., "Methods For Accelerating Dynamic Durability Tests". 9th International Conference on Recent Advances in Structural Dynamic, Southampton, UK, 2006.

[8] Su H., "Automotive CAE Durability Analysis Using Random Vibration Approach", Advanced Technology Office, Visteon Corporation, 2005. 
[9] Murat A., Mehmet Ç. "Vibration Fatigue Analysis and Multi-axial Effect in Testing of Aerospace Structures", Mechanical Systems and Signal Processing 23897 -907, Ankara, Turquia, 2009. 